\title{
Design of a Videogame to Explore Morality
}

\author{
Sarah Hodge \\ Bournemouth University \\ Talbot Campus, Fern \\ Barrow \\ Poole, BH12 5BB \\ shodge@ \\ bournemouth.ac.uk
}

\author{
Jacqui Taylor \\ Bournemouth University \\ Talbot Campus, Fern \\ Barrow \\ Poole, BH12 5BB \\ jtaylor@ \\ bournemouth.ac.uk
}

\author{
John McAlaney \\ Bournemouth University \\ Talbot Campus, Fern \\ Barrow \\ Poole, BH12 5BB \\ jmcalaney@ \\ bournemouth.ac.uk
}

\author{
Christos Gatzidis \\ Bournemouth University \\ Talbot Campus, Fern \\ Barrow \\ Poole, BH12 5BB \\ cgatzidis@ \\ bournemouth.ac.uk
}

\begin{abstract}
A number of video games involve moral narratives or require the player to make moral decisions and research from psychologists has helped to understand the effects video game content can have on how individuals behave. Recent research has started to examine the role of morality in video games: however, there are many inconsistencies in the findings. We propose that some of these inconsistencies could be due to using commercial video games for research purposes, which contain biases such as familiarity with the game and favourite characters. The process of playing video games requires consideration of Human Computer Interaction ( $\mathrm{HCl}$; i.e., how the game is designed and then how it is received by the player. The aim of this poster is to highlight the work being conducted to design a game in order to investigate how moral decisions are made in video games. The design of video games usually draws on an understanding of $\mathrm{HCl}$ to produce play that is entertaining and engaging for the player. The game development process in this research draws on a fusion of psychology and $\mathrm{HCl}$, and by drawing on theories of morality the design of the game will be grounded in psychology, as well as entertainment. Through fusing video game design principles, $\mathrm{HCl}$ and psychology, this work is novel in terms of a methodological as well as theoretical contribution to the area.
\end{abstract}

Keywords: video games, morality, design, game development, $\mathrm{HCl}$.

\section{INTRODUCTION}

Much-publicised concerns have been raised in the past about the moral content of video games such as the Grand Theft Auto series (Rockstar, 19972015), which includes nudity, prostitution, guns, drug dealing and driving recklessly. Other video games, such as Fallout 3 (Bethesda-Softworks, 2008), contain moral decisions to be made by the player with differing consequences for game success. The design of these games draws on psychological and $\mathrm{HCl}$ understanding to motivate and entertain. Thomas (2004) notes the different level of involvement, agency and interactivity of videogames, compared to other media. Agency is linked to the level of control or choice players have in video games, e.g. the difference between watching someone kill a character and the individual taking action by pressing a button to kill the character. This poster publication will identify how psychology and principles of $\mathrm{HCl}$ are used to design a game to explore moral decision-making. In doing so, it represents a fusion of game design, $\mathrm{HCl}$ and psychology.

\section{PSYCHOLOGY AND MORALITY IN VIDEO GAMES}

Research has started to investigate how an individual's offline sense of morality might relate to morality whilst playing video games. Weaver and Lewis (2012) examined the game Fallout 3 (Bethesda-Softworks, 2008) and found moral decisions in a video game were similar to moral decisions made in real life. However, Grizzard et al. (2016) used Call of Duty (Activision, 2009) and Operation Flashpoint (Bohemia-Interactive, 2001) and found that repeated exposure reduced feelings of guilt during game play. These inconsistencies could be due to methodological issues with using commercial video games as these contain biases (such as familiarity with the game and favourite characters). In addition, the morality programmed in video games has been described as being absolute, utilitarian and focusing on the action (Heron and Belford, 2014). These choices tend to include reward structures which can be juvenile and explicit. They also tend to be binary and, in some cases, ternary.

Recently, Joeckel et al. (2013) developed a game to examine the role of morality using the Moral 
Foundations Theory (MFT) (Haidt and Joseph, 2004). The game was made using the Aurora engine. The authors found that increased moral salience in the video game decreased the moral violations made. This was replicated in a similar study, with the additional finding that enjoyment did not influence moral salience (Joeckel et al., 2013). Developing a new video game did reduce some of the biases, however, there were limitations; the scenarios were text-based and the player was asked if another character should violate a scenario rather than the player violating a scenario themselves, thus the agency could have been an issue. This current project aims to draw on psychology and game development; in order to create a game to measure morality. This includes creating a stronger sense of moral agency and, at the same time, to produce an experience similar to what game participants would normally expect to have from gameplay.

\subsection{The development of the game}

The process of playing video games requires consideration by the game designer of the interaction between the player and the role they are playing. Thus, for the development of this new game, we aim to produce a game which is the enabling platform for research that is grounded in theoretical psychology and games design principles. A literature review was conducted to identify theories of morality relevant for application within a video game. MFT was chosen as it has been used previously in research (Joeckel et al., 2012, Joeckel et al., 2013) and scenarios could be developed from the moral themes. These moral themes include: Care/Harm, Fairness/Cheating, Loyalty/Betrayal, Authority/Subversion and Sanctity/Degradation (Haidt and Joseph, 2004, Haidt and Joseph, 2007). A sixth foundation is currently being developed, that of Liberty/Oppression. Clifford et al. (2015) developed scenarios from the MFT and these scenarios were then used to generate new scenarios for the game, based on the authors' scenarios. These scenarios are currently being piloted to check the validity of each one; each scenario is specific to one theme (foundation) and cannot overlap with other themes. However, Liberty/Oppression has been difficult to separate from the other themes, such as Fairness/Cheating. To try to resolve this, follow-up studies were conducted to examine the overlap and the scenarios were adjusted accordingly.

Once piloting is complete, the scenarios will be transferred and programmed into the game using either the Unreal 4 or Unity game engines (both contemporary tools for game creation). These game engines will construct the virtual environment, where the moral decisions will be made. The game will then be played by participants and their moral decisions will be recorded and analysed. It is intended that the game will appear similar to the real world and reasonably photorealistic for believability and will compare video game choices from real life morality. The design and interface of the game will be similar to a first person Role Playing Game (RPG). The game will use usability principles to produce an easy-touse offering that does not require previous experience of video games. In addition to this, a small tutorial will be sufficient for the learnability of the game. Rewards and motivational techniques will be used cautiously, but, in order to acknowledge the importance of the users' experience and expectation of entertainment, $\mathrm{HCl}$ principles based on affective computing will be employed. The aim of this is that the game is still an engaging and entertaining experience and similar to a typical, commercial first person RPG.

University students will be observed and recorded playing the game. Before and after playing the game, participants will be given the Moral Foundations Questionnaire (Graham et al., 2008) to measure real life morality, in comparison to video game decisions. Other measures collected will include the level of engagement felt when playing the game.

\subsubsection{Conclusion}

In summary, by incorporating $\mathrm{HCl}$ principles and a psychological understanding of morality into the design of a game we will explore the interaction between morality and video game playing. The biases inherent in commercial games will be reduced and a controlled virtual environment will be used to test alternative scenarios. This research builds on previous theoretical research in psychology, while also applying psychological understanding and $\mathrm{HCl}$ principles to game design. The empirical findings will have implications for further research and practice on the incorporation of moral education through gamification.

\section{REFERENCES}

Activision 2009. Call of Duty: Modern Warfare 2 [Video game].

Bethesda Softworks 2008. Fallout 3 [Video game].

Bohemia Interactive 2001. Operation Flashpoint: Cold War Crisis. [Video game].

Clifford, S., Iyengar, V., Cabeza, R. \& SinnottlArmstrong, W. 2015. Moral foundations vignettes: a standardized stimulus database of scenarios based on moral foundations theory. Behavior research methods, 1-21.

Graham, J., Haidt, J. \& Nosek, B. 2008. The Moral Foundations Questionnaire (MFQ) [Online]. Available: http://www.moralfoundations.org/ questionnaires (Accessed July 2015). 
Grizzard, M., Tamborini, R., Sherry, J. L. \& Weber, R. 2016. Repeated Play Reduces Video Games' Ability to Elicit Guilt: Evidence from a Longitudinal Experiment. Media Psychology, 1-24.

Haidt, J. \& Joseph, C. 2004. Intuitive ethics: How innately prepared intuitions generate culturally variable virtues. Daedalus, 133, 55-66.

Haidt, J. \& Joseph, C. 2007. The moral mind: How five sets of innate intuitions guide the development of many culture-specific virtues, and perhaps even modules. The innate mind, 3, 367-391.

Heron, M. J. \& Belford, P. H. 2014. Do You Feel Like a Hero Yet? Externalised Morality in Video Games. Journal of Games Criticism, 1, 1-22.

Joeckel, S., Bowman, N. D. \& Dogruel, L. 2012. Gut or Game? The Influence of Moral Intuitions on Decisions in Video Games. Media Psychology, 15, 460-485.
Joeckel, S., Bowman, N. D. \& Dogruel, L. 2013. The Influence of Adolescents' Moral Salience on Actions and Entertainment Experience in Interactive Media. Journal of Children and Media, 7, 480-506.

Rockstar 1997- 2015. Grand Theft Auto (GTA). New York [Video game].

Thomas, N. 2004. Video Games as Moral Universes. Topia (York University), 101-115.

Weaver, A. J. \& Lewis, N. 2012. Mirrored Morality: An Exploration of Moral Choice in Video Games. CyberPsychology, Behavior \& Social Networking, 15, 610-614. 